\title{
Spatial Working Memory and the Brainstem Cholinergic Innervation to the Anterior Thalamus
}

\author{
Anna S. Mitchell, John C. Dalrymple-Alford, and Michael A. Christie \\ Christchurch Movement Disorders and Brain Research Group Psychology Department, University of Canterbury, \\ Christchurch, New Zealand 8001
}

\begin{abstract}
The anteroventral thalamic nucleus (AV) has a role in spatial memory, but the influence of the prominent brainstem cholinergic projection to this region is unknown. Here, spatial memory in a 12-arm radial maze was examined after $0.15 \mu$ l bilateral AV infusions of scopolamine. In part one, rats visited six arms singly (the phase $1 \mathrm{arms}$ ) and, after a 10 min delay, were allowed free choice to both phase 1 arms and the remaining six baited arms (phase 2 arms). Scopolamine (10 $\mu \mathrm{g})$ administered during the delay increased errors to both phase 1 and phase 2 arms, whereas PBS infusions increased phase 1 arm errors only. The PBS effect was the result of inserting the internal cannulas alone and not the infusion. The same dose of scopolamine $(10 \mu \mathrm{g})$ infused before maze testing (part two: no phase 1 arms, no delay) also impaired spatial memory over and above the effects of both PBS and no-infusion, which did not differ
\end{abstract}

markedly. Part two also showed that choice latency and choice strategies were unaffected by PBS and scopolamine. Cannulation and infusion procedures in both parts one and two did not produce any negative carryover effects across multiple control (no internal cannula) sessions, and a trypan blue manipulation indicated that infusions were restricted to the $\mathrm{AV}$ region. This study provides the first direct evidence that the brainstem cholinergic innervation to the limbic thalamus influences learning and memory, which may have important implications for human neurological conditions such as alcohol-related disorders and schizophrenia.

Key words: anterior thalamic nuclei; cholinergic systems; laterodorsal tegmental nucleus; scopolamine; spatial working memory; alcohol disorders; schizophrenia
The anterior thalamic nuclei (ATN) are important limbic structures that play an essential role in learning and memory. Aggleton and Brown (1999) proposed that the hippocampal formation, with the fornix, ATN, mammillary bodies, and retrosplenial cortex, constitute a distributed neural axis responsible for spatial and context-dependent memory. Neuroanatomical considerations suggest that the ATN occupy a unique, pivotal position within this "extended hippocampal system" (Aggleton and Sahgal, 1993). Indeed, rats with ATN lesions show substantial spatial memory deficits, especially in the radial maze (Aggleton et al., 1996; Byatt and Dalrymple-Alford, 1996; Sziklas and Petrides, 1999). Other studies have revealed that ATN neurons show increased $c$-fos activation in intact rats and decreased $c$-fos activation after fornix lesions when spatial memory is challenged in the radial maze (Vann et al., 2000a,b).

One interesting feature of the ATN region is that it exhibits an intense acetylcholinesterase stain. ATN neurons do not synthesize choline acetyltransferase, but they express cholinergic heteroreceptors and receive one of the highest numbers of cholinergic terminals in the thalamus (van Groen et al., 1993). These cholinergic terminals derive predominantly from laterodorsal tegmental nucleus (LDTg) efferents (Hallanger et al., 1987; Sikes

\footnotetext{
Received Aug. 13, 2001; revised Nov. 28, 2001; accepted Dec. 6, 2001.

This research was approved by the Animal Ethics Committee of the University of Canterbury. We gratefully acknowledge grant support from the Department of Psychology, University of Canterbury.

Correspondence should be addressed to Anna S. Mitchell, Christchurch Movement Disorders and Brain Research Group, Department of Psychology, University of Canterbury, Private Bag 4800, Christchurch, New Zealand 8001. E-mail: asm43@student.canterbury.ac.nz.

Copyright (C) 2002 Society for Neuroscience $\quad 0270-6474 / 02 / 221922-07 \$ 15.00 / 0$
}

and Vogt, 1987; Shibata, 1992). Unlike the involvement of forebrain cholinergic systems in learning and memory, surprisingly little is known of any similar role for the LDTg brainstem cholinergic system (for review, see Everitt and Robbins, 1997). Figure $1 A$ summarizes the main connections between the ATN, LDTg, and mammillary bodies. Electrophysiological evidence suggests that LDTg efferents to the ATN regulate mammillothalamic terminals in a facilitatory manner (Pare and Steriade, 1990). An increase in muscarinic receptor binding in the anteroventral (AV) component of the ATN has also been reported during active avoidance learning in rabbits (Vogt et al., 1991). Consequently, Gabriel (1993) suggested that coactivation of mammillothalamic and tegmental efferents produce the changes in ATN neural activity that parallels the progression of avoidance learning.

The lack of direct evidence on the influence of the brainstem projection to the ATN is perhaps partly attributable to the previous uncertainty of the involvement of the ATN itself in mnemonic processes (Greene and Naranjo, 1986; Beracochea et al., 1989) and partly the obvious technical difficulties of targeting this subcortical region. Given the renewed interest in the ATN and its functional association with the hippocampus, we assessed the influence of cholinergic neurotransmission in the AV region on spatial working memory in the radial maze by using local microinfusion of the muscarinic antagonist scopolamine administered via an unconventional angle of approach. The greatest density of the brainstem cholinergic innervation to the ATN occurs predominantly ipsilaterally to the AV nucleus, with weaker and almost no projections to the anteromedial and anterodorsal thalamic nuclei, respectively (Hallanger et al., 1987; Shibata, 1992; Bentivoglio et al., 1993). Importantly, it is also known that lesions restricted to the AV nucleus are sufficient to produce markedly impaired radial 
maze performance (Aggleton et al., 1996; Byatt and DalrympleAlford, 1996).

\section{MATERIALS AND METHODS}

\section{Subjects}

The study used female hooded rats, 9 months old at the time of surgery (180-240 gm). Rats were housed either three or four per cage preoperatively and individually after surgery. They were maintained under a reversed light schedule (off from 7:00 A.M to 7:00 P.M.), at $80-85 \%$ of normal free-feeding weight during behavioral testing. Just before surgery, rats had access to food ad libitum, but their weights were gradually reduced again during postoperative recovery $(14 \mathrm{~d})$. Testing occurred between 8:00 A.M. and 7:00 P.M. at a rate of six to seven sessions per week, with one session per day. All rats were involved in both parts one and two of the present experiment.

\section{Surgery}

Anesthetized rats $(50 \mathrm{mg} / \mathrm{ml}$ sodium pentobarbital at $1.65 \mathrm{ml} / \mathrm{kg}, 20 \mathrm{~min}$ after $0.125 \mathrm{mg} / \mathrm{ml}$ atropine sulfate at $1.5 \mathrm{ml} / \mathrm{kg}$, i.p.) were placed in a double-arm stereotaxic apparatus (David Kopf Instruments, Tujunga, $\mathrm{CA}$ ), with the incisor bar set $7.5 \mathrm{~mm}$ below the interaural line rather than a more traditional orientation. Bilateral 22 gauge stainless steel cannula guides (Plastics One Inc., Roanoke, VA) were implanted above the AV in the transverse plane of the stereotaxic apparatus at $10^{\circ}$ angles from the midline and were secured to the cranium with four jeweler screws and dental acrylic. This combination of a downward-tilted head and a $10^{\circ}$ lateral angle for stereotaxic placement of the guide cannulas created a double-angled orientation for these guides. To facilitate placement of the guides, they were implanted together with 28 gauge internal cannulas (2 $\mathrm{mm}$ projection), and the latter were replaced with a dummy obdurator (flush with end of guide) when surgery was complete. To improve the accuracy of guide cannula placements in any given rat, it was also necessary to use stereotaxic anteroposterior (AP) coordinates that varied with the horizontal stereotaxic distance between lambda and bregma (L-B) observed during surgery. Four L-B distance criteria (in $\mathrm{cm}: 0.60$ and $0.61 ; 0.62$ and $0.63 ; 0.64,0.65$, and 0.66 ; or 0.67 and 0.68 ), sufficient to incorporate all of the L-B distances observed, were used to select one of four corresponding AP target coordinates (respectively, -0.245 , $-0.255,-0.265$, or $-0.275 \mathrm{~cm}$ posterior to bregma). That is, the AP coordinate was allowed to vary between -0.245 and $-0.275 \mathrm{~cm}$ across rats. In all cases, the lateral coordinates were -0.259 (left) and +0.254 (right) $\mathrm{cm}$, to compensate for a bias when working with the angled guides. The single depth from dura used for the internal cannula was ventral $(\mathrm{V})-0.52 \mathrm{~cm}$ (i.e., guides set at $\mathrm{V}-0.32 \mathrm{~cm}$ ). During surgery, one rat sustained an accidental lesion to the substantia innominata and was excluded. Some rats were lost before completion of testing because they showed signs of illness or their cannulas became insecure.

\section{Histology}

On completion of the experiment, rats received a lethal dose of sodium pentobarbital, followed with bilateral infusions of a $4 \%$ trypan blue solution (Sigma Chemicals, Castle Hill, New South Wales, Australia) as per the microinfusion procedure below. Rats were intracardially perfused $(0.9 \%$ saline, followed by $4 \%$ formalin), and brains were fixed in $4 \%$ formalin for $24 \mathrm{hr}$ before transfer to a long-term glucose solution. Frozen coronal sections throughout the anterior thalamus were cut at 50 $\mu \mathrm{m}$ with alternate sections stained with cresyl violet and acetylcholinerease to locate the sites of the internal cannula tips.

\section{Microinfusion}

A small volume $(0.15 \mu \mathrm{l})$ of scopolamine hydrobromide (Sigma Chemicals) dissolved in PBS, $\mathrm{pH} 7.36$, was infused bilaterally at a rate of 0.05 $\mu \mathrm{l} / \mathrm{min}$ for a $3 \mathrm{~min}$ period using 28 gauge internal cannula that extended $2 \mathrm{~mm}$ below the guides. Each internal cannula was attached via connectors and plastic tubes to a $1.00 \mu$ l Hamilton syringe driven by a motorized microinfusion pump (Bioanalytical Systems Inc., West Lafayette, IN) and remained in situ for an additional 3 min period after drug infusions to ensure their diff usion. Based on a $\log 10$ scale, doses of scopolamine at $1.00,2.51,6.31,10.00$, and $15.85 \mu \mathrm{g}$ were diluted from a stock solution of $20.00 \mu \mathrm{g} / 0.15 \mu \mathrm{l}$ PBS.

\section{Radial maze testing}

Apparatus. Rats were trained on an elevated ( $85 \mathrm{~cm}$ above floor) 12 -arm radial maze, with a $35-\mathrm{cm}$-wide central wooden hub painted black and equally spaced aluminum arms $(9 \times 65 \mathrm{~cm})$. Each arm had 3 -cm-high borders and a single Perspex barrier $(25 \times 20 \mathrm{~cm})$ adjacent to the hub. A black wooden insert $(8.5 \times 5 \times 3 \mathrm{~cm})$ at the end of each arm incorporated a food well $(2 \mathrm{~cm}$ diameter, $1 \mathrm{~cm}$ deep) with $2 \times 0.1 \mathrm{gm}$ pieces of chocolate when baited plus inaccessible chocolate present below the well at all times. Clear Perspex guillotine doors that could be raised singly or as one unit via overhead cables governed access to each arm.

Procedure. Before surgery, all rats received familiarization with the maze and training to run to the food wells for reinforcement. Chocolate pieces were scattered throughout the maze for the first two sessions when cage mates were able to explore the whole maze freely. Then individual rats received familiarization in the maze with the doors closed for a $5 \mathrm{sec}$ confinement each time a rat returned to the central hub before making an additional arm visit. In addition, the chocolate pieces were moved progressively farther along the arms across five sessions until being present only in the food wells for an additional seven presurgery familiarization sessions. After surgery, three more familiarization sessions followed by all the test sessions were conducted in a new and different room after 2 weeks of postoperative recuperation. During postoperative testing, groups of up to 13 rats were kept in a large covered holding box $(63 \times$ $63 \times 27 \mathrm{~cm}$ ) adjacent to the test room before their daily training session to minimize any effects of individual housing (which may impair radial maze performance) (Einon, 1980).

Two separate radial maze tasks were used (part one and part two), in which all of the rats participated. Previous radial maze work on the effects of systemically administered scopolamine has suggested that more reliable radial maze deficits occur when a delay procedure is used (Buresova and Bures, 1982; Bolhuis et al., 1988). Hence, part one of the experiment involved testing in a two-phase radial maze task, with the two phases separated by a delay. Scopolamine was administered at the beginning of the delay, and performance in the second phase (choice phase after the delay) was examined. Various doses were administered, together with an assessment of the effects of the cannulation procedure itself (i.e., simply inserting the internal cannulas alone). Then part two of the experiment used a standard version of the radial maze task (i.e., no delay, no forced choice), with scopolamine administered just before testing. Throughout testing during both parts one and two, rats were given one radial maze test session per day at approximately the same time each day and were confined for $5 \mathrm{sec}$ to the central wooden hub between choices to prevent the adoption of simple response strategies that might otherwise minimize spatial memory (Olton, 1987; Byatt and Dalrymple-Alford, 1996).

Part one: testing using a delay radial maze task. On each daily session, the rat was initially allowed to visit only single arms, evenly distributed around the maze, until six individual baited arms had been visited (i.e., six sequential "errorless"-forced choice visits; phase 1). Phase 1 was followed by a delay, and then the rat was given free choice of all 12 arms (phase 2) to find the remaining six nonvisited arms that were still baited. Rats were confined to the maze center after every arm visit in both phases. For phase 1, eight configurations of six forced choices were counterbalanced across rats per session, and these configurations were rotated across sessions within animals. For the first 10 training sessions, a $20 \mathrm{sec}$ delay occurred between phase 1 and phase 2, whereas from the 11th session onward, either a $10 \mathrm{~min}$ or a $20 \mathrm{sec}$ delay alternated between phases. These alternating sessions allowed rats to familiarize to the longer delay that was used when administering the infusions, while continuing acquisition of the two-phase task. At the beginning of each 10 min delay, the rat was always removed by the experimenter from the last arm visited in phase 1 and held in a towel while its obdurators were removed and replaced again. During the remainder of the 10 min delay, the rat was held in a cage in the room adjacent to the testing room before its return to the central hub of the maze. Although there was no time limit imposed during phase 1 , testing in phase 2 was terminated when the rat had entered the remaining six baited arms, once an additional $7 \mathrm{~min}$ had elapsed, or the rat made an additional 24 arm visits. Rats received their first infusions on reaching an initial performance criterion for phase 2 performance, invoked after a minimum of five $10 \mathrm{~min}$ delay sessions and within a maximum of eleven 10 min delay sessions. This criterion required the rat to retrieve the remaining six rewards within 12 visits on three consecutive sessions, and all rats retained for analysis were performing well within this criterion before any infusions. During all drug-testing periods, an infusion-free session (no cannula insertion) was always run between any infusion conditions. On rare occasions, an additional infusion-free session was run if the rat took $>12$ visits to 
retrieve the remaining six reinforcements. Infusions during part one were made at the beginning of a 10 min delay between the sixth and seventh arm visits and finished $\sim 7$ min before reentering the maze.

Three different assessments were made during part one: one betweengroup assessment and two within-group assessments. Initially, the cohort of rats was separated into four randomly allocated independent groups. Because of the novelty of making infusions of scopolamine into the AV, and to check that $\mathrm{AV}$ infusions produced only temporary effects on behavior, this initial assessment divided the cohort into four randomly selected groups $(n=4-6)$. Three groups received an infusion on two sessions (interspersed with a $20 \mathrm{sec}$ delay infusion-free session) of only one of three doses of scopolamine $(1.00,2.51$, or $6.31 \mu \mathrm{g})$, and the fourth group received PBS only. Second, an assessment of the effects on performance of the cannulation procedure (i.e., simply inserting the internal cannulas alone) was conducted. This second assessment involved all rats receiving two different conditions counterbalanced across consecutive sessions of testing. One condition involved the insertion of the internal cannulas alone into the guides (but no infusion) as per the cannulation procedure (sham infusion); the other condition involved a 10 min delay only. The third and key assessment in part one involved all rats receiving bilateral infusions of three higher doses of scopolamine $(6.31,10.00$, and $15.85 \mu \mathrm{g}$ ) and PBS across four daily sessions (interspersed with $10 \mathrm{~min}$ delay infusion-free sessions). The order of administering infusions throughout this third assessment was based on a Latin square design to counterbalance the order of conditions across rats.

Part two: testing in a standard (no delay) radial maze task. On completing part one of the experiment $\sim 7$ weeks after surgery, all rats received an uninterrupted session in a standard radial maze task to retrieve all 12 baits at the outset (i.e., no delay). The training involved a rat freely selecting an arm with all doors raised each time, after a 5 sec confinement to the central hub on returning from a previous arm visit as before. After this initial session, which showed that all rats readily performed the new version of the task with few errors, counterbalanced bilateral inf usions of scopolamine at $10.00 \mu \mathrm{g}$ or PBS were administered for all rats ending 7 min before the daily session in the maze. An infusion-free (no delay) session was run between these two sessions. A daily session was complete in part two once the rat had retrieved all 12 rewards, had made a total of 24 arm visits, or $10 \mathrm{~min}$ had elapsed.

\section{RESULTS}

\section{Histology}

The presence of internal cannula tips located bilaterally in the AV was set as a strict histology criterion for inclusion in data analysis. Twenty-one rats with appropriate bilateral internal cannula tip placements satisfied this criterion (with six exclusions). The internal cannula tip placements for these 21 rats are shown in Figure $1 B$. Dispersion of all trypan blue dye infusions was restricted to the anterior thalamic nuclei in both hemispheres, with only a small dispersion to the reticular thalamic nuclei (Fig. $1 C$ ). The double-angled guide placements allowing a $2 \mathrm{~mm}$ internal projection were successful in minimizing damage to adjacent structures. In successful implants, the guide cannulas produced slight damage toward the posterior dorsolateral fimbria-fornix region, with more obvious damage through the most rostral part of the hippocampal formation. The passage of the internal cannulas produced only minor damage to the fimbria-fornix above the ATN and, depending on the position of the tract, either slight damage to the posterior anterodorsal nucleus or the most rostral aspect of the laterodorsal nucleus. Three of the rats that failed the inclusion criterion had cannula tip placements located in the fimbria-fornix and lateral ventricle. Internal cannula tip placements for the other three exclusions were such that only unilateral and/or partial infusions would have reached the AV region, with the contralateral infusions going directly into the fimbria-fornix and ventricles.
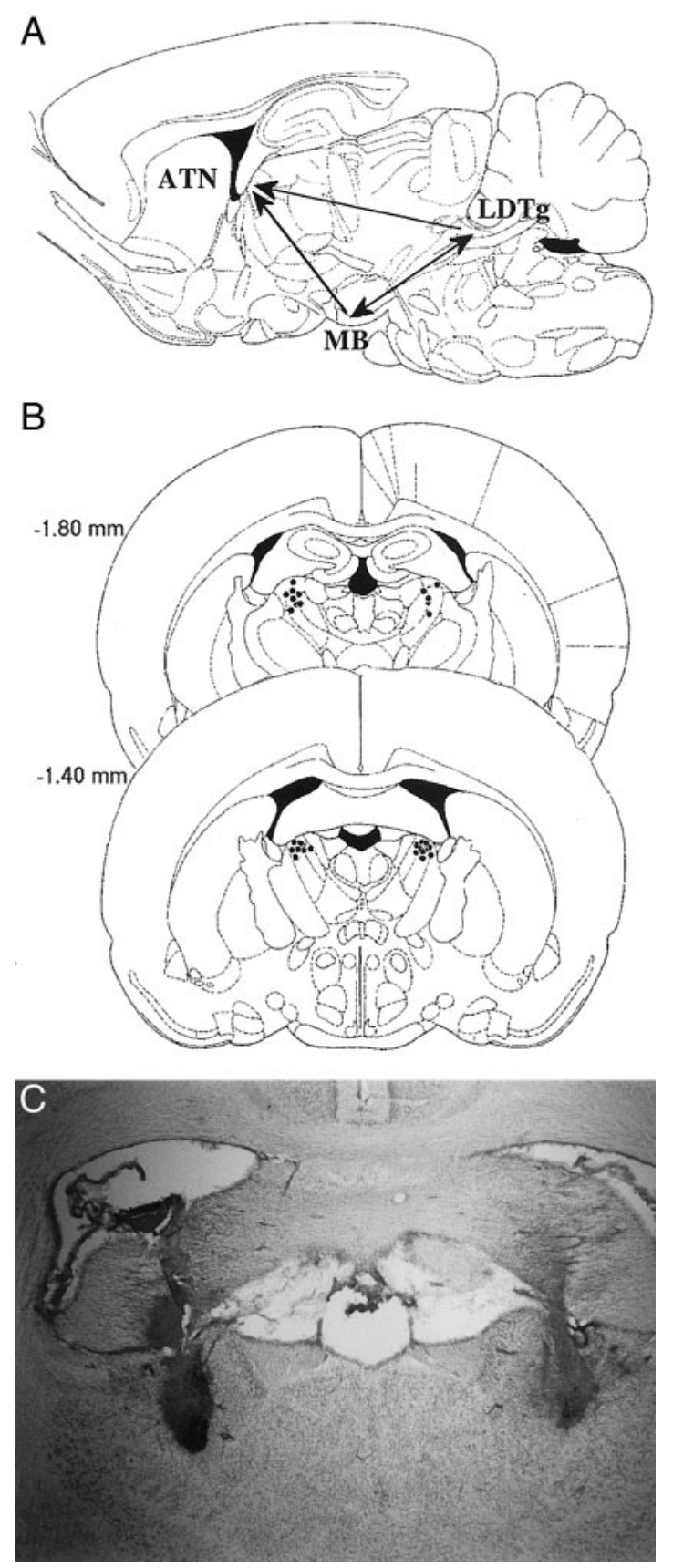

Figure 1. A, Schematic of the brainstem laterodorsal cholinergic system projections to the anterior thalamic nuclei and the main connections between these structures and the mammillary body region. $M B$, Mammillary bodies. $B$, Approximate bilateral locations from bregma of the internal cannula tips (black circles) for all rats used in the final data analysis. Most location sites were located intermediate to these two plates. Note that many overlapping cannula tips are not shown [adapted from Paxinos and Watson (1986)]. C, Photomicrograph example of trypan blue dye infusion to the AV (rat 79). Note that evidence of the trypan blue dye solution in the cannula track does not indicate the same for scopolamine infusions because a $3 \mathrm{~min}$ dispersion period was used in the latter.

\section{Part one: working memory using the delay radial maze task}

The delay procedures produced three main findings. First, cannulation and infusion procedures during the 10 min delay did not produce any negative carryover effects on performance in subsequent control (no internal cannula) $20 \mathrm{sec}$ or $10 \mathrm{~min}$ delay 


\begin{tabular}{|c|c|c|c|c|c|}
\hline & No cannula & PBS & Scop $6.31 \mu \mathrm{g}$ & Scop $10.00 \mu \mathrm{g}$ & Scop $15.85 \mu \mathrm{g}$ \\
\hline Arm visits before an error & $3.91 \pm 0.29$ & $2.05 \pm 0.48$ & $1.45 \pm 0.39$ & $1.40 \pm 0.35$ & $1.25 \pm 0.38$ \\
\hline Mean total number of errors & $2.04 \pm 0.29$ & $4.50 \pm 0.87$ & $4.70 \pm 0.64$ & $6.10 \pm 0.93$ & $5.20 \pm 0.77$ \\
\hline $\begin{array}{l}\text { Errors to first six phase } 1 \text { arms } \\
\quad \text { (during phase } 2 \text { ) }\end{array}$ & $1.48 \pm 0.22$ & $3.70 \pm 0.67$ & $3.50 \pm 0.50$ & $4.35 \pm 0.60$ & $3.75 \pm 0.53$ \\
\hline $\begin{array}{l}\text { Errors to remaining six baited } \\
\text { phase } 2 \text { arms (during phase } 2 \text { ) }\end{array}$ & $0.56 \pm 0.14$ & $0.80 \pm 0.30$ & $1.20 \pm 0.27$ & $1.75 \pm 0.39$ & $1.45 \pm 0.29$ \\
\hline
\end{tabular}

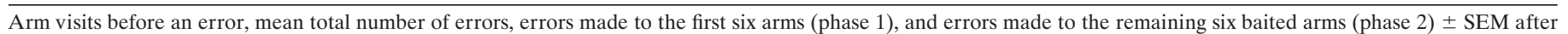

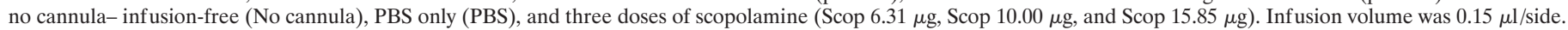

sessions. Second, PBS inf usions impaired performance, but this was probably attributable to the acute disruption effects of internal cannula insertion and not the infusion. More importantly, scopolamine increased errors to both phase 1 and phase 2 arms, whereas PBS only increased phase 1 arm errors.

It will be recalled that the first assessment in part one examined four groups of rats $(n=4-6)$ that received two separate bilateral inf usions of only one dose of either scopolamine at 1.00, 2.51, or $6.31 \mu \mathrm{g}$, or PBS vehicle only. These conditions did not affect the number of errors made or the number of arm visits before an error $\left(F_{(3,17)}<1.0\right)$. Performance was clearly more accurate on $20 \mathrm{sec}$ delay infusion-free sessions interspersed with the infusion sessions. All infusions disrupted performance [PBS vehicle only, $7.00 \pm 3.41$ (mean $\pm \mathrm{SD}$ ); scopolamine at $1.00 \mu \mathrm{g}, 5.00 \pm 1.07$; scopolamine at $2.51 \mu \mathrm{g}, 6.50 \pm 3.68$; and scopolamine at $6.31 \mu \mathrm{g}$, $8.60 \pm 4.30$ ], albeit only temporarily, compared with inf usion-free sessions $(1.12 \pm 1.06)$. Before any infusions, rats made only a few more errors after the $10 \mathrm{~min}$ delay than after the $20 \mathrm{sec}$ delay $(3.13 \pm 1.36$ and $2.14 \pm 1.58$, respectively).

The second assessment was a within-group analysis of the effects of inserting the internal cannula alone after the cannulation procedure but with no actual infusion. This manipulation showed that cannula insertion per se caused errors in performance $(5.57 \pm 3.20)$ when compared with the 10 min delay only (i.e., no internal cannula; $2.28 \pm 2.88$ ). Consequently, there was a significant main effect of condition $\left(F_{(1,20)}=13.25 ; p<0.002\right)$, although there was no significant effect of order of testing $\left(F_{(1,19)}=\right.$ 2.13; $p<0.16)$ and no order by condition interaction $\left(F_{(1,19)}<1.0\right)$.

The third assessment of the two-phase task used a within-group analysis of bilateral infusions of three higher doses of scopolamine $(6.31,10.00$, and $15.85 \mu \mathrm{g})$ compared with the effects of PBS infusion and no cannula-inf usion-free conditions (all conditions used a 10 min delay). To be included in the data analysis, rats had to make at least six arm visits (the minimum possible to retrieve the remaining baits) during phase 2 of all testing sessions $(n=20)$. One rat was excluded from the third assessment because it made only five arm visits after administration of scopolamine at $15.85 \mu \mathrm{g}$. In this third assessment, we analyzed the number of arm visits before an error, the total number of errors made, and the type of working memory errors made, such as errors to arms previously visited in phase 1 and revisits in phase 2 to arms remaining baited after phase 1 (Table 1 ).

Analysis of the mean number of arm visits before an error revealed a significant effect of condition $\left(F_{(4,76)}=8.68 ; p<\right.$ 0.0001). Newman-Keuls post hoc comparisons indicated that, during the no cannula-infusion-free condition, rats visited more arms before an error than across all four infusion conditions. There was also a significant main effect of condition for the total number of errors made $\left(F_{(4,76)}=6.40 ; p<0.0002\right)$, with all inf usion conditions producing more errors than the no cannulainfusion-free condition. Similarly, the number of errors made to arms that had been visited during phase 1 ("phase 1 arm errors") revealed a significant main effect of condition $\left(F_{(4,76)}=6.55 ; p<\right.$ $0.0001)$, again indicating that all types of infusion resulted in more errors to phase 1 visited arms relative to the no cannulainfusion-free condition. Of greater interest, however, was the finding that there was also a significant main effect of condition for the number of errors (i.e., revisits) made to arms that had remained baited after phase 1 ("phase 2 arm errors") $\left(F_{(4,76)}=\right.$ 3.44; $p<0.02$ ), because in this instance scopolamine at $10.00 \mu \mathrm{g}$ significantly increased phase 2 errors relative to the no cannulainfusion-free condition $(p<0.04)$ and produced a marginally nonsignificant increase compared with the PBS condition $(p<$ 0.06; Newman-Keuls comparisons). It is especially notable that PBS infusion had no effect relative to the no cannula-infusionfree condition on these phase 2 arm errors.

\section{Part two: working memory in a standard (no delay) radial maze task}

Based on results of the drug infusions during part one, scopolamine at $10.00 \mu \mathrm{g} / 0.15 \mu \mathrm{l}$ was used for bilateral infusions during part two. The number of arm visits before an error and total errors made provided the main measures for analysis. In addition, choice latencies and patterns of responding were also analyzed. To be included in the analysis, rats had to have made at least 12 arm visits during a session (the minimum possible to retrieve all baits). Four rats were excluded as they made only 4, 5, 8 and 11 arm visits after administration of scopolamine at $10.00 \mu \mathrm{g}$ (errors for these rats: $0,0,3$, and 2 , respectively).

In part two, clear and significant deficits in performance were revealed when infusions of scopolamine at $10.00 \mu \mathrm{g}$ were made before the daily session in the standard (no delay) radial maze task. The mean number of errors made $(n=17)$ (Fig. 2A) revealed a significant main effect of condition $\left(F_{(2,32)}=7.73 ; p<\right.$ 0.002). Newman-Keuls post hoc comparisons showed that, after infusion of scopolamine at $10.00 \mu \mathrm{g}$, the number of errors increased markedly relative to both no cannula-infusion-free and PBS conditions ( $p<0.002$ and $p<0.02$, respectively), but the latter two conditions did not differ $(p<0.15)$. In addition, there was no main effect or interaction for the order of testing ( $F$ values $<1.0$ ).

Analysis of the mean number of arm visits before an error also revealed a significant effect of condition $\left(F_{(2,32)}=16.58 ; p<\right.$ 0.0001) (Fig. 2B). Post hoc comparisons indicated that, during the no cannula-infusion-free condition, the number of arm visits made before an error was higher than the number of arms visited after PBS infusions $(p<0.02)$ and higher again relative to scopolamine at $10.00 \mu \mathrm{g} / \mathrm{side}(p<0.0002)$. In addition, however, 

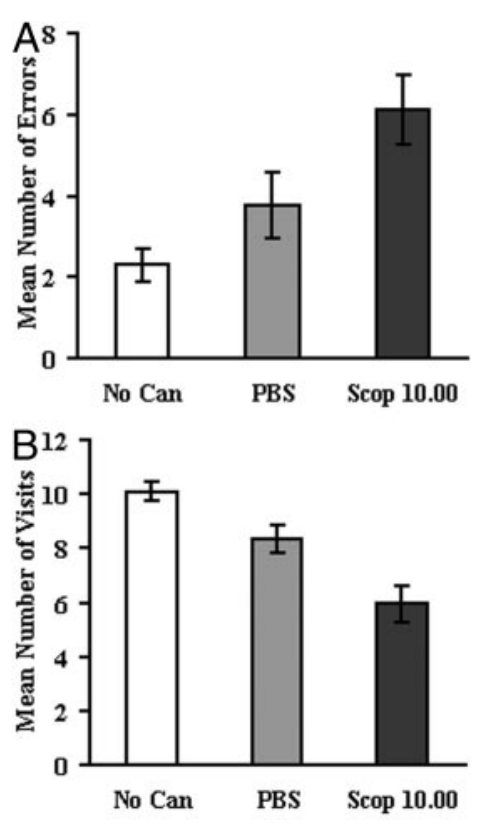

Figure 2. Part two: standard radial maze task (no delay). $A$, Mean \pm SEM number of errors. $B$, Mean number of arm visits before an error. No $C a n$, No internal cannula inserted-inf usion-free session; $P B S$, PBS vehicle only; Scop 10.00, scopolamine at $10.00 \mu \mathrm{g} / \mathrm{side}$.

the number of arms visited before an error was also higher after inf usions of PBS relative to scopolamine at $10.00 \mu \mathrm{g}(p<0.002)$.

The mean choice latencies were shorter for correct than incorrect arm choices (Fig. 3A,B). Analysis of the mean choice latencies for correct and incorrect arm visits across the three conditions indicated there was no significant difference of condition in either correct $(F<1.0)$ or incorrect $\left(F_{(2,32)}=1.65\right.$; NS $)$ choices. Thus, although four rats experienced some adverse reaction to the scopolamine infusion and failed to run the maze properly, activity in the remaining rats was not adversely affected.

Finally, Figure $3 C$ shows the pattern of choice responses made by rats during part two. Choices were analyzed across the first 12 choices based on the distance of the new choice from the arm just exited (collapsed across clockwise and counterclockwise choices). If a rat chose the same arm on a subsequent choice, the score was 0 . If the rat chose the adjacent arm, the score was 1 . If the rat chose the opposite arm, the score was 6 . Clearly, the rats did not adopt a simple response strategy such as choosing the adjacent arms, unlike the typical pattern found in radial maze tasks that do not use confinement by doors between choices (Higashida and Ogawa, 1987). Instead, the choices made by rats in the current study were more evenly distributed across choices 1 through 6 away from the arm just exited, although choices 4 and 5 were generally most preferred and choice 6 (opposite to last arm) was least preferred $\left(F_{(5,80)}=3.99 ; p<0.003\right)$ (choice 0 not included in the analysis). Importantly, these choice distributions did not change markedly across the no cannula-infusion-free, PBS, and scopolamine at $10.00 \mu \mathrm{g} / \mathrm{side}$ conditions. There was neither an overall effect of condition $\left(F_{(2,32)}=1.69\right.$; NS; with no significant Newman-Keuls differences) nor a condition by choice interaction $(F<1.0)$.

\section{DISCUSSION}

The present study provides the first demonstration that scopolamine infused directly into an anterior thalamic nucleus impairs
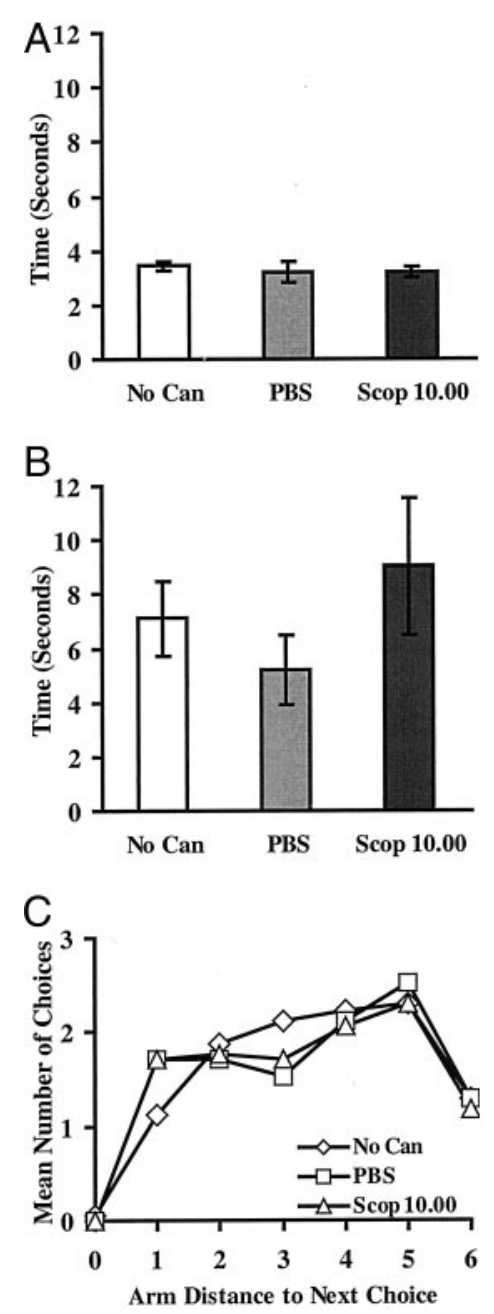

Figure 3. Part two: standard radial maze task (no delay). $A$, Mean \pm SEM choice latencies for correct arm visits. $B$, Mean choice latencies for incorrect arm visits. $C$, Response patterns: relative frequency of selecting arms at various distances (combined clockwise and counterclockwise) from the arm just exited. No Can, No internal cannula inserted-inf usionfree session; PBS, PBS vehicle only; Scop 10.00, scopolamine at 10.00 $\mu \mathrm{g} / \mathrm{side}$.

spatial working memory in rats and the first explicit evidence that the ascending LDTg cholinergic projection to the limbic thalamus influences memory processes. Bilateral inf usions into the AV of a small volume of scopolamine, at $10.00 \mu \mathrm{g} / \mathrm{site}$, produced increased errors during 12-arm radial maze testing. This effect was evident to some degree compared with the two control conditions in the delay version of the task (part one: phase 2 arm errors). A clear impairment, however, was evident in the standard maze procedure (part two) in which it was also shown that there were negligible effects on nonspecific behavioral processes such as choice pattern and response latency. Direct scopolamine infusions to hippocampal targets also affect spatial memory regardless of the use of any delay (Buhot et al., 1995). Interestingly, $10.00 \mu \mathrm{g}$ of scopolamine was also the optimal dose that impaired spatial memory after infusions to the medial prefrontal cortex (Ragozzino and Kesner, 1998). Our findings complement and extend previous work that suggested that brainstem-ATN efferents are involved in learning and memory (Pare and Steriade, 1990; Vogt et al., 1991; Gabriel, 1993).

PBS infusions also impaired spatial working memory, but this 
effect was evident when discriminating spatial locations that had already been visited before a delay (part one: phase 1 arm errors) rather than for newer locations only recently visited (part one: phase 2 arm errors; and part two). The different type of error produced by PBS infusions is consistent with the conclusion that specific scopolamine effects in the AV region caused the behavioral problems observed with this muscarinic antagonist. Moreover, the negative effect of PBS infusions was the result of the cannula insertion rather than the infusion, which reflects the technical difficulty of successfully targeting the limbic thalamus. The AV lies immediately inferior to the fornix. It seems likely that the internal cannula effect was caused by an acute but temporary disruption to the fimbria-fornix above the ATN and either the posterior anterodorsal nucleus or the most rostral aspect of the laterodorsal thalamus. Although unusual, our use of a $2 \mathrm{~mm}$ internal cannula projection coupled with a double angle for the guide implant meant that we successfully minimized any permanent structural damage to any critical overlying structures. Satisfactory cases showed relatively little evidence of damage to the fornix and the most rostral part of the dorsal hippocampus, beyond some unavoidable minor cannula damage. The success of our unconventional cannula approach was confirmed by the fact that, despite being familiarized preoperatively with the maze in one test environment and then tested postoperatively in a new one, rats quickly achieved baseline accuracy for spatial memory performance, which they maintained throughout the study, despite delay testing and multiple infusions. In contrast, our pilot work with a unilateral AV lesion and a contralateral cannula implant produced permanent maze impairments, as did bilateral guide cannulas that extended to the dorsal surface of the AV.

There are several reasons to believe that our scopolamine effects were primarily related to localized events in the AV. We have no direct evidence that the scopolamine infusions did not also affect adjacent structures, but extremely small infusion volumes were used and the trypan dye injections in rats with appropriate implants indicated that these infusions remained primarily within the AV. There was no indication of any dispersion to the mediodorsal thalamus. A few cases indicated that some dye, and presumably also scopolamine, spread to the adjacent reticular thalamic nuclei, but the laterality of the cannula placements was not related to behavioral performance. Neither the mediodorsal nucleus nor the reticular nuclei appear to be important for spatial working memory (Aggleton and Brown, 1999; Wilton et al., 2001).

The radial maze is a key task that has been used to evaluate the effects of scopolamine on spatial working memory. Together with evidence from abundant lesion work, it has been assumed that the effects of intraperitoneal administration of scopolamine on spatial memory are primarily a reflection of its influence on basal forebrain projections and their frontal and limbic targets (Blokland, 1995; Everitt and Robbins, 1997). This view has been reinforced by intracerebral infusion work with scopolamine aimed at the hippocampal and corticolimbic targets of the basal forebrain cholinergic system (Buhot et al., 1995; Everitt and Robbins, 1997; McIntyre et al., 1998; Ragozzino and Kesner, 1998; Mishima et al., 2000). Our findings show that the ascending brainstem cholinergic system to the AV should also be considered a candidate in work on systemically administered cholinergic drugs. Although it is possible that some basal forebrain efferents contribute to the cholinergic projection in the $\mathrm{AV}$, the balance of evidence strongly implicates the LDTg as the primary, if not the sole, source of this projection (Sikes and Vogt, 1987; Shibata, 1992; Bentivoglio et al., 1993).

The current study reinforces the importance of the ATN within a proposed extended hippocampal system responsible for spatial and event memory (Aggleton and Brown, 1999). In particular, it emphasizes a unique role for the ATN independently of any direct hippocampal system intervention. The temporary impairment on working memory in the radial maze after blockade of cholinergic neurotransmission in the AV provides new pharmacological evidence of the importance of this region in learning and memory processes. This evidence is consistent with previous reports that spatial memory in the radial maze task is accompanied by neuronal activation in the AV nucleus (Vann et al., 2000a) and is sensitive to damage within the AV component of the ATN in particular (Aggleton et al., 1996; Byatt and Dalrymple-Alford, 1996).

Knowledge that the brainstem cholinergic system modulates the ATN during spatial memory processing supports the inclusion of the LDTg in neuroanatomical models of event memory (Gabriel, 1993). The ATN occupy a key position within the extended hippocampal system, with dense reciprocal connections to different layers of the subicular complex and the cingulate and retrosplenial cortices (Aggleton and Sahgal, 1993; Shibata, 1993, 1998; van Groen et al., 1993; Vogt, 1993). Allocortical input to the ATN is both direct via the fornix and indirect via the mammillary bodies. These neural pathways constitute a hippocampal limbic loop responsible for the expression of context-dependent and spatial components of event memory. Our findings, and evidence that the responsiveness of ATN cells to direct and indirect hippocampal data is probably facilitated by the LDTg projection to the ATN (Pare and Steriade, 1990), show that this thalamic region is regulated by input from the ascending brainstem cholinergic system. It is also possible that these cholinergic brainstem efferents modulate limbic system loops in general. Limbic system loops regulate the significance of highly processed sensory information, which is used by frontal neuronal systems to govern the expression of behavior (Braak and Braak, 2000). The medial prefrontal cortex, in turn, projects to the LDTg (Groenewegen and Uylings, 2000).

The present findings have important implications for human brain disorders. The relative influence of different components of the limbic thalamus on learning and memory is uncertain, but increasing animal evidence suggests that the ATN play a more prominent role in episodic memory processes than do the mediodorsal nuclei (Aggleton and Sahgal, 1993; Byatt and Dalrymple-Alford, 1996; Aggleton and Brown, 1999; Sziklas and Petrides, 1999) (but see Gaffan and Parker, 2000). The association between the ATN and diencephalic amnesia, alcohol, and the Korsakoff syndrome is receiving increasing interest (Belzunegui et al., 1995; Kopelman, 1995; Harding et al., 2000), but any interaction between the ATN and its cholinergic afferents in these conditions has yet to be addressed. Similarly, schizophrenic patients also exhibit remarkably poor episodic memory (Aleman et al., 1999), which may to some degree be related to their neurobiological deficits in the limbic thalamus (Hazlett et al., 1999; Young et al., 2000), including the AV region (Danos et al., 1998). Problems with the integrity of the tegmental cholinergic system have also been reported in schizophrenia, although the nature of this disruption is disputed (Karson et al., 1993, 1996; German et al., 1999). We showed that muscarinic blockade of the ascending brainstem cholinergic innervation to the AV, which arises predominantly from the LDTg, impairs performance in an animal 
analog of human episodic memory. Clearly, these various lines of evidence suggest that future research will need to assess the prominent brainstem laterodorsal tegmental-thalamic cholinergic interactions with greater interest.

\section{REFERENCES}

Aggleton JP, Brown MW (1999) Episodic memory, amnesia, and the hippocampal-anterior thalamic axis. Behav Brain Sci 22:425-489.

Aggleton JP, Sahgal A (1993) The contribution of the anterior thalamic nuclei to anterograde amnesia. Neuropsychologia 31:1001-1019.

Aggleton JP, Hunt PR, Nagle S, Neave N (1996) The effects of selective lesions within the anterior thalamic nuclei on spatial memory in the rat. Behav Brain Res 81:189-198.

Aleman A, Hijman R, de Haan EHF, Kahn RS (1999) Memory impairment in schizophrenia: a meta-analysis. Am J Psychiatry 156:1358-1366.

Belzunegui T, Insausti R, Ibanez J, Gonzalo LM (1995) Effects of chronic alcoholism on neuronal nuclear size and neuronal population in the mammillary body and the anterior thalamic complex of man. Histol Histopathol 10:633-638.

Bentivoglio M, Kultas-Ilinsky KI, Ilinsky I (1993) Limbic thalamus: structure, intrinsic organisation and connections. In: Neurobiology of cingulate cortex and limbic thalamus (Vogt BA, Gabriel M, eds), pp 71-122. Boston: Birkhauser.

Beracochea DJ, Jaffard R, Jarrard LE (1989) Effects of anterior or dorsomedial thalamic ibotenic lesions on learning and memory in rats. Behav Neural Biol 51:364-376.

Blokland A (1995) Acetylcholine: a neurotransmitter for learning and memory? Brain Res Rev 21:285-300.

Bolhuis JJ, Strijkstra AM, Kramers RJK (1988) Effects of scopolamine on performance of rats in a delayed-response radial maze task. Physiol Behav 43:403-409.

Braak H, Braak E (2000) Pathoanatomy of Parkinson's disease. J Neurol 247 [Suppl 2]:II/3-II/10.

Buhot MC, Patra SK, Naili S (1995) Spatial memory deficits following stimulation of hippocampal 5-HT1B receptors in the rat. Eur J Pharmacol 285:221-228.

Buresova O, Bures J (1982) Radial maze as a tool for assessing the effect of drugs on the working memory of rats. Psychopharmacology (Berl) $77: 268-271$.

Byatt G, Dalrymple-Alford JC (1996) Both anteromedial and anteroventral thalamic lesions impair radial-maze learning in rats. Behav Neurosci 110:1335-1348.

Danos P, Baumann B, Bernstein HG, Franz M, Stauch R, Northoff G, Krell D, Falkai P, Bogerts B (1998) Schizophrenia and anteroventral thalamic nucleus: selective decrease of parvalbumin-immunoreactive thalamocortical projection neurons. Psychiatry Res 82:1-10.

Einon D (1980) Spatial memory and response strategies in rats: age, sex and rearing differences in performance. Q J Exp Psychol 32:473-489.

Everitt BJ, Robbins TW (1997) Central cholinergic systems and cognition. Annu Rev Psychol 48:649-684.

Gabriel M (1993) Discriminative avoidance learning: a model system. In: Neurobiology of cingulate cortex and limbic thalamus (Vogt BA, Gabriel M, eds), pp 478-576. Boston: Birkhauser.

Gaffan D, Parker A (2000) Mediodorsal thalamic function in scene memory in rhesus monkeys. Brain 123:816-827.

German DC, Manaye KF, Wu D, Hersh LB, Zweig RM (1999) Mesopontine cholinergic and non-cholinergic neurons in schizophrenia. Neuroscience 94:33-38.

Greene E, Naranjo JN (1986) Thalamic role in spatial memory. Behav Brain Res 19:123-131.

Groenewegen HJ, Uylings HBM (2000) The prefrontal cortex and the integration of sensory, limbic and autonomic information. In: Cognition, emotion and autonomic responses: the integrative role of the prefrontal cortex and limbic structures (Uylings HBM, Van Eden CG, De Bruin JPC, Feenstra MGP, Pennartz CMA, eds), pp 3-28. Elsevier: Amsterdam

Hallanger AE, Levey AI, Lee HJ, Rye DB, Wainer BH (1987) The origins of cholinergic and other subcortical afferents to the thalamus in the rat. J Comp Neurol 262:105-124.

Harding A, Halliday G, Caine D, Kril J (2000) Degeneration of anterior thalamic nuclei differentiates alcoholics with amnesia. Brain 123:141-154

Hazlett EA, Buchsbaum MS, Byne W, Wei TC, Spiegel-Cohen J, Geneve C, Kinderlehrer R, Haznedar MM, Shihabuddin L, Siever LJ (1999) Three-dimensional analysis with MRI and PET of the size, shape, and function of the thalamus in the schizophrenia spectrum. Am J Psychiatry 156:1190-1199.

Higashida A, Ogawa N (1987) Difference in the acquisition process and the effect of scopolamine on radial maze performance in three strains of rats. Pharmacol Biochem Behav 27:483-489.

Karson CN, Casanova MF, Kleinman JE, Griffin WST (1993) Choline acetyltransferase in schizophrenia. Am J Psychiatry 150:454-459.

Karson CN, Mrak RE, Husain MM, Griffin WST (1996) Decreased mesopontine choline acetyltransferase levels in schizophrenia. Mo Chem Neuropathol 29:181-191.

Kopelman MD (1995) The Korsakoff syndrome. Br J Psychiatry 166:154-173.

McIntyre CK, Ragozzino ME, Gold PE (1998) Intra-amygdala infusions of scopolamine impair performance on a conditioned place preference task but not a spatial radial maze task. Behav Brain Res 95:219-226.

Mishima K, Iwasaki K, Tsukikawa H, Matsumoto Y, Egashira N, Abe K, Egawa T, Fuijwara M (2000) The scopolamine-induced impairment of spatial cognition parallels the acetylcholine release in the ventral hippocampus in rats. Jpn J Pharmacol 84:163-173.

Olton DS (1987) The radial arm maze as a tool in behavioral pharmacology. Physiol Behav 40:793-797.

Pare D, Steriade M (1990) Control of the mammillothalamic axis by brainstem cholinergic laterodorsal tegmental afferents: possible involvement in mnemonic processes. In: Brain cholinergic systems (Steriade $\mathrm{M}$, Bieso D, eds), pp 337-354. Oxford: Oxford UP.

Paxinos G, Watson C (1986) The rat brain in stereotaxic coordinates, Ed 2. San Diego: Academic.

Ragozzino ME, Kesner RP (1998) The effects of muscarinic cholinergic receptor blockade in the rat anterior cingulate and prelimbic/infralimbic cortices on spatial working memory. Neurobiol Learn Mem 69:241-257.

Shibata H (1992) Topographic organization of subcortical projections to the anterior thalamic nuclei in the rat. J Comp Neurol 323:117-127.

Shibata H (1993) Efferent projections from the anterior thalamic nuclei to the cingulate cortex in the rat. J Comp Neurol 330:533-542.

Shibata H (1998) Organization of projections of rat retrosplenial cortex to the anterior thalamic nuclei. Eur J Neurosci 10:3210-3219.

Sikes RW, Vogt BA (1987) Afferent connections of anterior thalamus in rats: sources and association with muscarinic acetylcholine receptors. J Comp Neurol 256:538-551.

Sziklas V, Petrides M (1999) The effects of lesions to the anterior thalamic nuclei on object-place associations in rats. Eur J Neurosci 11:559-566.

van Groen T, Vogt BA, Wyss JM (1993) Interconnections between the thalamus and retrosplenial cortex in the rodent brain. In: Neurobiology of cingulate cortex and limbic thalamus (Vogt BA, Gabriel M, eds), pp 123-150. Boston: Birkhauser.

Vann SD, Brown MW, Aggleton JP (2000a) Fos expression in the rostral thalamic nuclei and associated cortical regions in response to different spatial memory tests. Neuroscience 101:983-991.

Vann SD, Brown MW, Erichsen JT, Aggleton JP (2000b) Using fos imaging in the rat to reveal the anatomical extent of the disruptive effects of fornix lesions. J Neurosci 20:8144-8152.

Vogt BA (1993) Structural organization of cingulate cortex: areas, neurons, and somatodendritic transmitter receptors. In: Neurobiology of cingulate cortex and limbic thalamus (Vogt BA, Gabriel M, eds), pp 19-70. Boston: Birkhauser.

Vogt BA, Gabriel M, Vogt LJ, Poremba A, Jensen E, Kubota Y, Kang E (1991) Muscarinic ${ }^{2}$ receptor binding increases in anterior thalamus and cingulate cortex during discriminative avoidance learning. J Neurosci 11:1508-1514.

Wilton LA, Baird AL, Muir JL, Aggleton JP (2001) Excitotoxic lesions of the rostral thalamic reticular nucleus do not affect the performance of spatial learning and memory tasks in the rat. Behav Brain Res 120:177-187.

Young KA, Manaye KF, Liang C, Hicks PB, German DC (2000) Reduced number of mediodorsal and anterior thalamic neurons in schizophrenia. Biol Psychiatry 47:944-953. 